\title{
A study of the controlling parameters of fuel air mixture formation for ECN Spray A
}

\author{
K. Vogiatzaki ${ }^{1 *}$, C. Crua ${ }^{1}$, R. Morgan ${ }^{1}$, M.R. Heikal ${ }^{1}$ \\ ${ }^{1}$ Advanced Engineering Centre, University of Brighton, Lewes road, Brighton BN2 4GJ, UK \\ ${ }^{*}$ Corresponding author: k.vogiatzaki@brighton.ac.uk
}

\begin{abstract}
Designing future ultra-high efficiency, ultra-low emission engines requires an in depth understanding of the multiscale, multi-phase phenomena taking place in the combustion chamber. The performance of the fuel delivery system is key in the air fuel mixture formation and hence the combustion characteristics, however in most spray modelling approaches is not considered directly. Thus, it is important to understand how the selection of models that mimic injection process affect predictions. In this paper we present an Eulerian-Lagrangian framework based on OpenFOAM libraries to model spray injection dynamics. The framework accounts for primary droplet formation (based on a parcel method with predefined initial droplet size distribution), secondary droplet breakup, evaporation and heat transfer. In order to account for the interaction of droplets with turbulence, simulations were performed within the LES context with two different turbulence models. A systematic variation of the key injection parameters (parcel number, parcel size distribution) of the parcel method as well as the grid size was considered. Varying the parcel number affects the initial droplet size distribution which in turn, depending on the selection of the turbulence and the evaporation sub-models, affects: spray dispersion; spray penetration; and subsequent droplet size distribution. Results were validated against the baseline experimental data for evaporating ECN Spray A with n-dodecane chosen as a surrogate for Diesel fuel.
\end{abstract}

\section{Keywords}

ECN Spray A, Eulerian-Lagrangian, LES, OpenFOAM

\section{Introduction}

In both Diesel and spark ignition engines fuel is injected into the combustion chamber at elevated pressures. Nowadays realistic injection pressures for common-rail Diesel reach up to 3500 bars. The reason behind the need for such extreme pressures is to promote primary and secondary atomisation until a combustible mixture is formed. The higher the injection pressure the higher the shear between the static air within the combustion cylinder and the liquid. At extreme ambient pressures and temperatures the fuel can even exhibit supercritical behaviour that causes a reduction in its surface tension. Combustion occurs in a lifted, turbulent diffusion flame mode. Numerous studies indicate that the combustion and emissions in such engines are strongly influenced by the lifted flame characteristics, which are in turn determined by fuel and air mixing in the upstream region of the lifted flame, and consequently by the liquid breakup and spray development processes $[1,19,14,5,6]$. These processes clearly play a critical role in determining the engine combustion and emission characteristics.

From a numerical standpoint simulating spray combustion in modern engines involves a number of challenges mostly associated with the multiphase, multi-scale nature of the phenomenon. Scales vary from the molecular level (reactions) to microns (droplets) to $\mathrm{mm}$ (turbulence) and to meters (combustor dimensions). Thus, 'all componentall scale' analysis with direct numerical simulations (DNS) is prohibitive with the current computational capabilities for real size combustors. Only a very limited number of studies have been performed in engines with DNS [18]. Reynolds Averaged Navier Stokes (RANS) [17] and more recently Large Eddy Simulation (LES) based approaches are typically employed for engine simulations [7, 3, 22, 12]. RANS, which is the industrial standard approach is based on ensemble averaged governing equations. Although numerically efficient and relatively accurate in predicting the qualitative behaviour of the sprays, RANS cannot predict the local unsteadiness in the mixing flow field. This is a considerable drawback considering that ultra-high injection pressures promote supersonic behaviour of the jet and locally the creation of shock waves that further promote unsteadiness [15]. Moreover, RANS does not allow the study of cycle-to-cycle variation phenomena relevant to the spray evolution that currently is a subject that attracts considerable interest, and is also linked to spray spatial and temporal variations. The LES approach, which is based on spatially filtered governing equations, can capture the large scale flow structures based on the filter size. However, the unresolved small-scale structures are still modelled, which makes LES dependent on the sub grid scale models used and the grid resolution. Since LES can capture local unsteadiness and is computationally more attractive than a DNS based approach, it has received significant attention in the past decade, especially for simulation of internal combustion engines.

An additional difficulty in the modelling of turbulent combustion in realistic engine geometries is that in the effort to reduce computational cost and grid complexity, in many of the existent approaches the injector is not simulated directly and the effects of the in-nozzle flow and primary atomisation are modelled indirectly. The existent models based on the so-called parcel method have considerable weaknesses, the most important of which is the fact that 
the injected (initial) droplet size distribution needs to be selected a-priory. If experimental data are not available then fine tuning is required, making the final results dependent on the degree of tuning. Moreover, these models are mostly tailored to intermediate injection pressures of equipment of the past as well as to the RANS context. Extending these models to the LES context, understanding the resulting challenges and suggesting modifications is an area of active research. The sensitivity of the predictions to the injection model also undermines efforts for better evaporation and combustion models since in sprays all the phenomena present are interlinked and the injection part is a key controlling parameter of the initial mixture formation. For instance, even if the evaporation model predicts accurately the droplet size reduction because of heat transfer, if the parcels initially injected represent droplets with sizes considerably smaller than the real ones, simulations of liquid penetration will fail because the droplets will evaporate considerably quicker.

In recent years, various studies were performed in both experimental $[9,14]$ and numerical front $[21,20,22,23]$ in an effort to better understand spray dynamics at ultra-high pressures. Within the experimental results several institutions have provided high-fidelity measurements of macroscopic spray parameters such as spray penetration, liquid length and vapor penetration as well combustion related parameters such as ignition delay, lift-off length, and soot emissions for a range of fuels, ambient and injection conditions. Such datasets can be accessed through the Engine Combustion Network [11].

\section{Experimental data}

In the current work we use experimental data from Sandia National Laboratories at operating conditions known as ECN 'Spray A' (see Table 1). A constant-volume, quiescent, pre-burn-type combustion vessel is used to generate high-temperature and high-pressure gases. A premixed combustible mixture is spark-ignited. The combustion products are cooled until they reach the desired pressure and temperature. Then the diesel fuel injector is triggered and fuel injection occurs. The conditions for $n$-dodecante used as a surrogate of diesel fuel. In Fig 1 the experimental pictures of Spray A at three differnt time instances obtained with different techniques is presetned. In pictures from both techniques the light blue line indicates the liquid penetration vs time [11]. The time instances were selected to correspond to the time instances that numerical results will be rpesented in the following secitons.

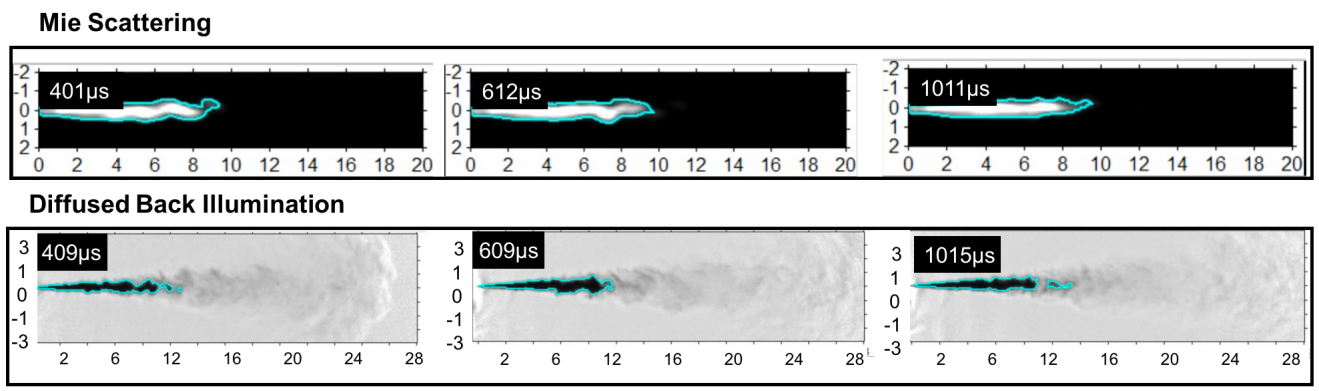

Figure 1. Experimental images of Spray $A$ at three different time instances obtained with different techniques. In pictures from both techniques the light blue line indicates the liquid penetration vs time [11].

\section{Numerical setup}

The numerical simulation of the two-phase flows is performed in the framework of an Eulerian-Lagrangian approach within an Open Source code (OpenFOAM) [8]. The governing equations were solved on two different grid sizes. For

Table 1. Summary of experimental conditions [11].

\begin{tabular}{c|c}
\hline Experimental conditions & n-dodecane \\
\hline \hline Ambient temperature $(\mathrm{K})$ & 900 \\
Ambient density $\left(\mathrm{kg} / \mathrm{m}^{2}\right)$ & 22.8 \\
Composition & $100 \% \mathrm{~N}_{2}$ \\
Injection pressure $(\mathrm{bar})$ & 1500 \\
Fuel temperature $(\mathrm{K})$ & 363 \\
Nozzle diameter $(\mu \mathrm{m})$ & 90 \\
Duration of injection $(\mathrm{ms})$ & 1.5 \\
Total mass injected $(\mathrm{mg})$ & 3.5 \\
Fuel density $\left(\mathrm{kg} / \mathrm{m}^{3}\right)$ & 750 \\
\hline
\end{tabular}


the rest of the paper we use the notation "Grid 1" for the coarser grid with average cell size of $0.5 \mathrm{~mm}$ and "Grid 2" for a finer grid with average cell size of $0.3 \mathrm{~mm}$. The time step is calculated based on the Courant number criterion: Comax $_{\max }=\frac{u \Delta t}{\Delta x}$ where $\Delta x$ is the grid size while $\Delta t$ is the time step. For the calculations presented in the results
section the $\mathrm{Co}_{\max }=0.1$.

\section{Turbulence model}

LES is based on the idea of computing the large, energy-containing eddy structures (filtered quantities) which are resolved on the computational grid, whereas the smaller, more isotropic, sub-grid structures (SGS) are modelled. The filter width is taken as the cube root of the local grid cell volume. The effect of the small scales is obtained through the sub-grid scale stress term $\left(\tau_{i j}^{s g s}=\widetilde{u_{i} u_{j}}-\widetilde{u}_{i} \widetilde{u}_{j}\right)$ that must be modelled. There are two popular types of turbulence models: a) algebraic eddy viscosity models in which the stress tensor $\tau_{i j}^{\text {sgs }}$ is related to the resolved strain rate tensor $\widetilde{S}_{i j}$ by means of a scalar eddy viscosity given by an algebraic equation; b) one-equation eddy viscosity models. Both model groups are based on the Boussinesq hypothesis associating $\tau_{i j}^{s g s}$ with a SGS turbulent viscosity $\mu_{T}$. However their main difference is that one-equation SGS models overcome the deficiency of local balance assumption between the SGS energy production and dissipation adopted in algebraic models. Such a phenomenon may occur in high Reynolds number flows and/or in the cases of coarse grid resolutions. In this paper we assess two models (one of each group): The Wall-Adapting Local Eddy-viscosity model (WALE) [13] which is an algebraic eddy viscosity models and the Kinetic Energy Model (KEM) [24] that belongs to the category of oneequation eddy viscosity models. The main difference between the proposed WALE model in comparison to other models of this group, is that the SGS viscosity is dynamically computed with the square of the velocity gradient tensor rather than the resolved strain rate used in Smagorinsky-type models that have been tested in previous work [21] for Spray A. This velocity tensor can not only account for the effects of both strain and rotation rate of the smallest resolved turbulence fluctuations, but also recover the proper near-wall scaling for the eddy viscosity without requiring dynamic procedure. Moreover, the WALE model is invariant to any coordinate translation or rotation and no test-filtering operation is needed, it is therefore considered well suitable for LES in complex geometries [13] as the ones in IC engines.

\section{Injection model}

As mentioned in the introduction one of the greatest challenges associated with the Eulerian-Lagrangian approach is modelling the near-nozzle flow. In this region a liquid core forms from the liquid fuel being injected through the injector. Ligaments are separated from this liquid core and form droplets that evaporate and mix with the ambient gas. When a combined Eulerian-Lagrangian framework is used then the fuel spray is treated as a dispersed liquid phase, which moves and interacts with the surrounding continuous gas phase. The spray is represented by an ensemble of discrete "parcels". Each parcel contains a number of droplets with the same size, velocity and temperature. Droplets in a parcel are considered as spherical, which is a rather strong assumption especially for regions close to the nozzle where ligaments instead of droplets are expected to be formed. The droplet parcels are tracked in a Lagrangian fashion as they move through the gas phase, exchanging mass, momentum and energy. The effect of the droplet parcels on the continuous phase due to drag, heat and mass transfer is implemented via source terms in the gas phase conservation equations.

Figure 2 shows a schematic of the injection process modelled in our current calculations. The injection model is a solid-cone injection model. The user supplies a drop diameter probability density function (PDF) with parameters. In our work we have examined two different models a) a Rosin-Rammler (RR) [2] with spreading parameter $n=3$ and mean diameter $\bar{d}=50 \mu \mathrm{m}$. The RR distribution function is based on the assumption that an exponential relationship exists between the droplet diameter, $d$, and the mass fraction $Y_{d}$ of droplets with diameter greater than $d: Y_{d}=e^{(d / \bar{d})^{n}}$ b) A fixed value distribution with mean droplet size $\bar{d}=90 \mu \mathrm{m}$. In this method all droplets injected have the same size and their size only changes as they move through the domain because of evaporation and secondary break up. One point that should be made is that for sprays with low initial velocity, the droplets can retain their sizes for quite a long period after the primary breakup. It is, therefore, essential to provide a correct droplet size distribution for fuel sprays of low injection velocity, such as the pressure-swirl type of gasoline injector. For diesel sprays of high initial velocity, the droplet size distribution is not expected to be as important to the final droplet distribution if an appropriate model for the secondary breakup is applied. The higher the initial velocity of the jet, the sooner the secondary breakup occurs and the lower the dependence of the final droplet sizes on the droplet size distribution of the primary breakup.

The velocity of the injected parcel is calculated as $u_{d}=\frac{\dot{m}}{C_{d} \rho A}$ where $\mathrm{A}$ is the area of the injection (defined by the diameter of the nozzle), $C_{d}$ is the discharge coefficient (=0.9) and $m \dot{a} s s$ is the mass flow rate. Within this model the velocity vector direction is defined by a random angle size within a limit (in our case $10^{\circ}$ ) which is a user-defined constant and does not depend on the droplet size which might lead to inaccuracies. It should be underlined that the mass (or volume) given initially to each parcel depends on the mass flow rate profile of the injector while the mean droplet size characterising the parcel depends on the initial distribution. This means that the number of droplets in each parcel is a statistical number which can vary from a fraction of a droplet to thousands of droplets depending also on the number of parcels used. The higher the number of parcels used the lower the number of droplets each 
parcel will contain.

It becomes evident that one of the most important parameters in this injection model is the number of parcels injected per second (PPS). The higher the PPS the more accurate is the representation of the initial pre-selected distribution. For the case of a fixed droplet size this is not particularly important since even a small size of parcels is enough for the statistical representation of the injection process while for the case of the RR the number of parcels is more important to faithfully reproduce the droplet size distribution.



Figure 2. Simulation details of the injection process including the domain size

\section{Parcel Tracking}

In Lagrangian spray simulations, the particles representing the liquid are moving in a fixed Eulerian framework as described above. Tracking them and defining the cells they go through are clearly important issues. In OpenFOAM the approach used is the face-to-face tracking. The process can be described in four steps [10]: 1) Initially the parcel is moved until it reaches a cell boundary or the entire time step if it remains in the same cell; 2) Then a check is performed to evaluate if the parcel changes cell; 3) The time it took to move out of the first cell is calculated, and the parcel properties are updates; 4) Following the momentum change to the cell that the parcel has been in are added. If the parcel still has time left to move we go back the the first step of the algorithm. Parcels tracked by face-to-face tracking cannot 'skip' cells, which improves the predictions of transfer of mass, momentum and energy.

\section{Secondary breakup}

The breakup model used is the Kelvin-Helmholtz-Rayleigh-Taylor (KHRT) model [16]. This model, along with the TAB model, is one of the most widely used in Lagrangian spray simulations today. The KHRT model was chosen here since previous studies (within the RANS context) have indicated its superior performance under Diesel conditions [4]. It should be mentioned that it is also possible to use the TAB model, but often in conjunction with some form of primary atomisation model. The TAB model tends to break up the droplets very rapidly. The KHRT model includes two modes of breakup: KH breakup, accounting for unstable waves growing on the liquid jet due to differences in velocity between the gas and liquid; and RT breakup, accounting for waves growing on the droplets' surface due to acceleration normal to the droplet-gas interface. The relative performance of the two models within the LES context needs to be also examined in a future study.

\section{Summary of Test Cases}

In Table 2 a summary of all the cases considered in this paper is provided.

\section{Results and discussion}

Figure 3 shows predicted and measured liquid spray penetration at different times after start of injection (ASOI) under non-reacting conditions for n-dodecane at an ambient temperature of $900 \mathrm{~K}$. Liquid penetration is defined as the axial location encompassing $97 \%$ of the injected mass at that instant in time. The first observation is that for Cases 1-3 that the RR is used as initial distribution the results show great sensitivity to the particle number. Case 3 (with the lower number of particles and thus the less accurate representation of the RR) shows closer agreement with the experiments. This can be considered an indication that the RR with the selected parameters might not be 
the optimum distribution for this spray condition. Moving to a finer grid it can be seen that rather surprisingly for both Cases 4 and 5, irrespectively of the number of parcels used a considerably higher liquid penetration is predicted in comparison to the coarser mesh. Also the predictions are less dependent on the PPS number. Differences can only be noticed when a considerably low number of PPS is used as in Case 6. Using though 50.000 PPS is not a reasonable number to represent any spray statistics and thus it is not considered in the liquid penetration predictions. An additional interesting point is that the turbulence model appears to play a considerable role. Case 1 and 3 are run with the same number of parcels as Case 7 and 8 as well as the same grid however the liquid penetration predicted is different. For Case 7 and 8 regardless of the number of parcels the predictions agree with the experiments while for Case 1 and 3 the predictions depend on the number of parcels. An additional case (Case 9 ) is run with the KEM model and with 20 million PPS. It can be seen that for this case since the initial distribution is different (a fixed mean diameter value for all the injected droplets is chosen) the predictions are slightly different than Cases 7 and 8 although still in reasonably good agreement with the experiments. Finally it should be mentioned that looking at pictures in Fig 1 for all cases the spray disperses less than in experiments and potentially an even higher grid resolution with modifications to the particle model is required.

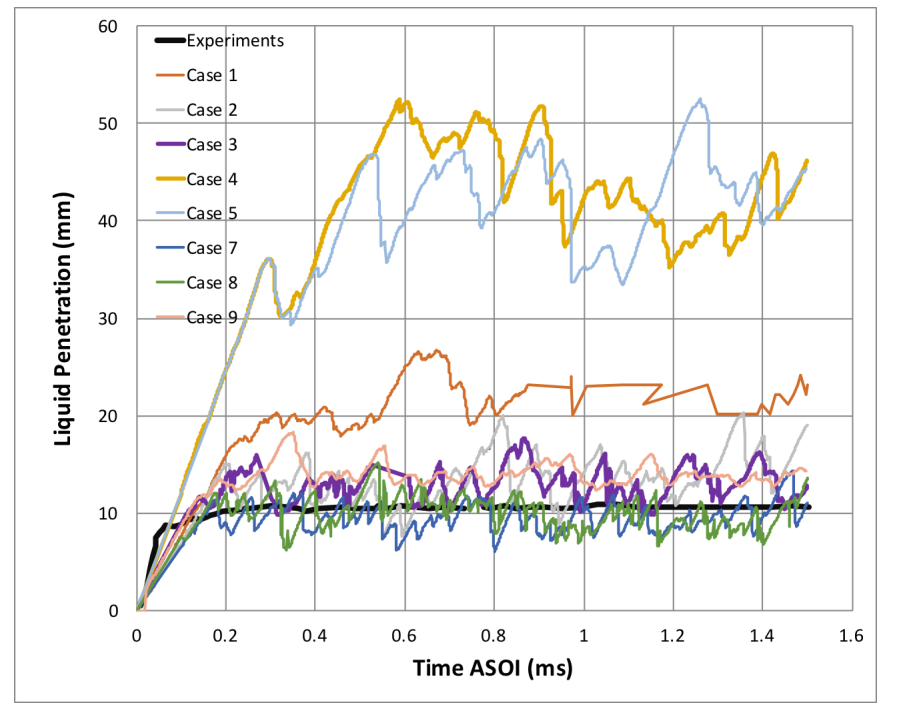

Figure 3. Measured [11] and predicted liquid penetration vs. time for the cases of Table 2

In order to get a better understanding of the conditions leading to these differences we include in our analysis Figs 4-6 that demonstrate the $\mathrm{C}_{12} \mathrm{H}_{26}$ (vapour) contour at two different time instances. We can see that in Fig 4 for the cases that use a rather large number of parcels $(20,000,000$ and 2,000,000) the results are similar. The length of the spray is similar while we can see that the vapour (indicated by the bright red areas of high $\mathrm{C}_{12} \mathrm{H}_{26}$ ) starts being formed even very close to the injection point. The behaviour is different in Fig 5 in which a higher grid resolution is used. The vapour diffuses less while it penetrates more. However the areas of high vapour concentration are at the tip of the spray and not close to the injection point. This behaviour is not compatible to what has been reported in the literature when different codes are used (see for example [21]). It should be underlined that the different grid sizes, apart from the direct effect they have on the turbulence resolution, also affect the parcel injection method because for the current calculations the time step is adjusted based on the Courant number. The average time step for Grid $1 \Delta t=2.5 \times 10^{-7} \mathrm{~s}$ while for Grid $2 \Delta t=1.3 \times 10^{-7} \mathrm{~s}$. The difference in the time step means that even for the cases that the same number of parcels per second is injected (for example Case 1 and 4 ) in reality a different number of parcels is injected per time step leading to a different representation of the initial droplet distribution PDF.

Table 2. Summary of numerical test cases.

\begin{tabular}{c|rccc}
\hline \hline Test Case & PPS & Grid Size $(\mathrm{mm})$ & Turbulence Model & Initial Distribution \\
\hline \hline Case 1 & $20,000,000$ & 0.5 & Wale & RR \\
Case 2 & $2,000,000$ & 0.5 & Wale & RR \\
Case 3 & 200,000 & 0.5 & Wale & RR \\
Case 4 & $20,000,000$ & 0.3 & Wale & RR \\
Case 5 & $2,000,000$ & 0.3 & Wale & RR \\
Case 6 & 50,000 & 0.3 & Wale & RR \\
Case 7 & $2,000,000$ & 0.5 & KEM & RR \\
Case 8 & 200,000 & 0.5 & KEM & RR \\
Case 9 & $20,000,000$ & 0.5 & KEM & Fixed Value \\
Case 10 & $20,000,000$ & 0.5 & KEM & RR \\
\hline
\end{tabular}


Moreover, the size of the time step is linked to the time that the particles interact with the underlying gas properties as explained in the Section "Parcel Tracking". For Grid 1 the average local Courant number is 0.0005 while for Grid 2 is 0.0009 . The difference in the local Courant number results because of the turbulence resolution locally which leads to a different local velocity. This implies that in reality for Grid 2 the particles remain less time in the cell, and thus they have less time to interact with the underlying Eulerian velocity field and exchange momentum which might explain why for the Grid 2 the spray penetrates much more than the other cases. Looking at Fig 6 we can see that when KEM turbulence model is used less sensitivity to the other parameters is noticed (PPS and initial distribution). The vapour penetration for all cases is similar however the distribution of the vapour is different. High vapour concentration (bright red areas) is seen through the spray for Case 7 while some isolated dense regions are noticed for Case 8. For Case 9 the behaviour is closer to Case 7 although a more uniform dispersion is noticed which might be attributed to the fact that less randomness is introduced in the inlet since all droplets have the same diameter.

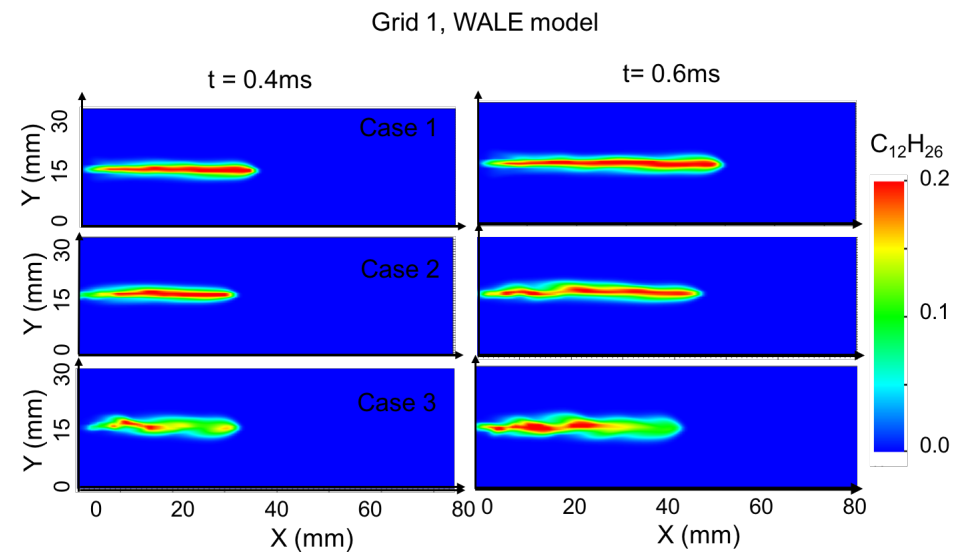

Figure 4. $\mathrm{C}_{12} \mathrm{H}_{26}$ contour plots for two different time instances using Grid 1 and WALE turbulence model.

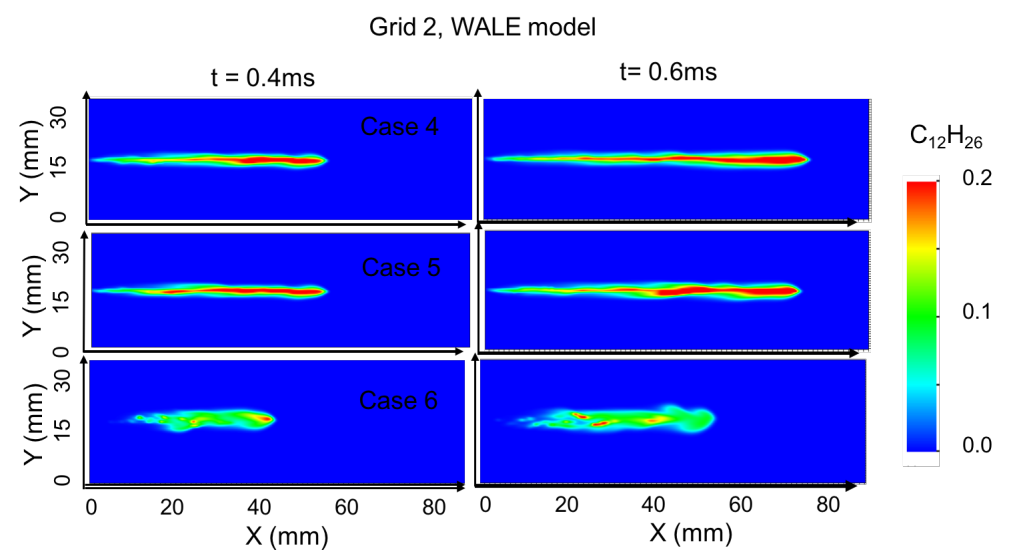

Figure 5. $\mathrm{C}_{12} \mathrm{H}_{26}$ contour plots for two different time instances using Grid 2 and WALE turbulence model.

Figure 7 shows the scatter plot of droplet diameter versus velocity magnitude (coloured by temperature) for the total number of the droplets in the domain at $t=3 \mathrm{~ms}$ for three different simulation cases. In all cases the PPS is 20 million. We will use this figure in order to get a better insight into the links between the predictions of the droplet size, the droplet velocity and the droplet temperature. For all cases the droplet size is mostly clustered in the range of $1-4 \mu \mathrm{m}$ and only fe parcels have diameters above $10 \mu \mathrm{m}$. Also we can see that in all cases droplets with smaller diameters have lower velocities while the droplets with larger diameters have higher velocities in some cases reaching up to $500 \mathrm{~m} / \mathrm{s}$. For Cases 1 and 4 the behaviour is similar although we can see that droplets with similar diameter for Case 1 have considerable lower velocity. For Case 4 there is a greater variation of the droplet velocity even for droplets with similar sizes. Droplets with small diameter $2-4 \mu \mathrm{m}$ have velocities ranging $200 \mathrm{~m} / \mathrm{s}-$ $300 \mathrm{~m} / \mathrm{s}$ and the temperature depends on the velocity. Droplets with higher velocities have also lower temperature since they have les tile to interact with the underlying flow field. For case 9 the behaviour is different and we can see a more linear relation between droplet size and velocity. Also we can see that for droplets in the range of 2-4 $\mu \mathrm{m}$ the temperatures are higher (above 550K)

Figure 8 shows the number of droplets vs droplet sizes for tho axila locations. Top raw is at $x=0.003 \mathrm{~m}$ (close to the injector) ant bottom raw at $x=0.09 \mathrm{~m}$ (close to the tip of the spray). In all cases the PPS is 20 million 
Grid 1, KEM model



Figure 6. $\mathrm{C}_{12} \mathrm{H}_{26}$ contour plots for two different time instances using Grid 1 and KEM turbulence model.
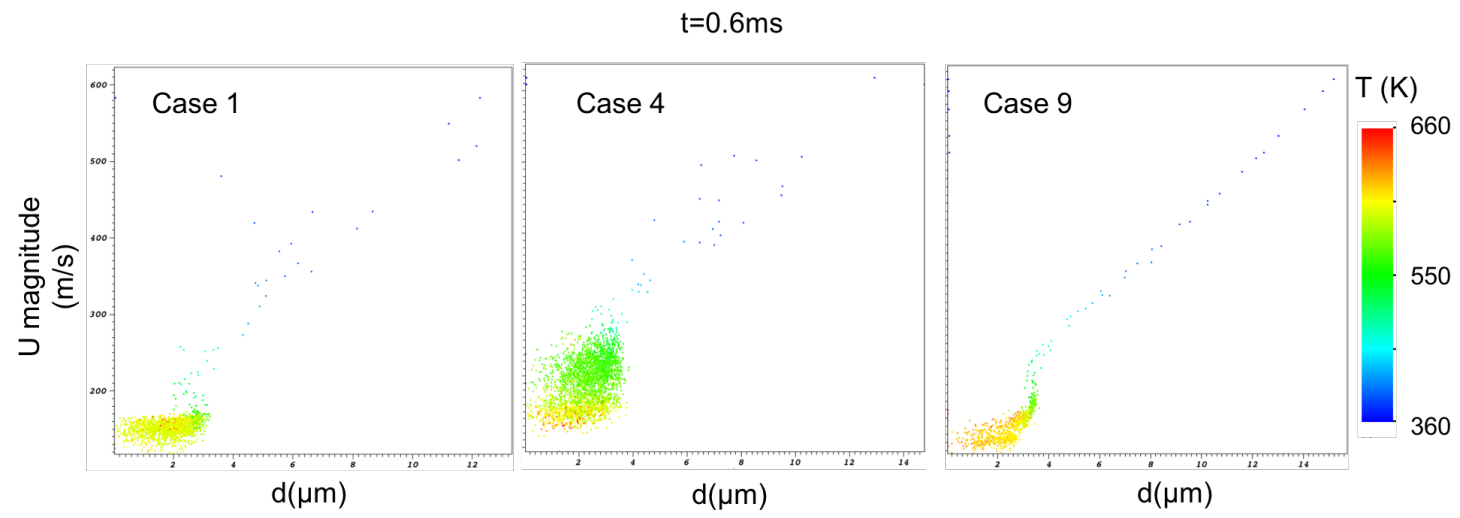

Figure 7. Scatter plot of droplet diameter versus velocity magnitude (coloured by temperature) for the total number of the droplets in the domain at $\mathrm{t}=3 \mathrm{~ms}$ for three different cases. In all cases the PPS is 20 million.

and the samples were taken for $t=0.6 \mathrm{~ms}$. It can be seen that close to the injector the droplet distribution as expected is different depending the selection of the initial distribution. When the RR distribution is used regardless of the turbulence selection model (for Case 1 Wale and for Case $10 \mathrm{KEM}$ ) the droplet distribution is similar. Much narrower distribution around droplet sizes of $3 \mu \mathrm{m}$ is noticed when a fixed value distribution is used (Case 9). Also it can be noticed that in both case the initial droplet size reduces rapidly. For example for Case 9 all droplets are injected with an average diameter of $90 \mu \mathrm{m}$ and after $3 \mathrm{~mm}$ their size has already reduced to $3 \mu \mathrm{m}$. Further downstream as expected the effect of the initial distribution reduces and all three cases predict an average droplet size of $2 \mu \mathrm{m}$. Moving from $3 \mathrm{~mm}$ to $9 \mathrm{~mm}$ the droplet radius reduction rate is smaller.

\section{Conclusions}

In this work we present an LES Eulerian-Lagrangian framework within OpenFOAM for the modelling of high pressure injection dynamics of ECN Spray A conditions. The framework accounts for primary droplet formation (based on a parcel method), secondary droplet breakup, evaporation and heat transfer. The sensitivity of the framework to different parameters that affect the predictions of the local mixture formation during breakup and evaporation is considered. Initially a systematic variation of the key injection parameters (parcel number, parcel size distribution) of the parcel method as well as the grid size is presented. Varying the parcel number affects the accuracy of the representation of the initial droplet size distributions, which in turn, depending on the selection of the initial droplet PDF, turbulence model and the evaporation model, affects a) spray dispersion b) spray penetration and c) downstream droplet size distribution. Moreover, two different turbulence models are considered. The selection of the turbulence model appears to be of high importance. For the cases considered the KEM model results are less sensitive to the other parameters (PPS, initial droplet PDF). Finally a rather unexpected discrepancy between the predictions of the coarse and the fine grid is noticed which is attributed to the algorithm for the parcel method and the adjustable time step used for the calculations. More detailed examination of the droplet diameter statistics including analysis of their radial distribution will be the subject of future work.

\section{Acknowledgements}

This work was supported by the UK's Engineering and Physical Science Research Council [grants EP/P012744/1 and EP/K020528/1]. 
20 million PPS, $\mathrm{t}=0.6 \mathrm{~ms}$
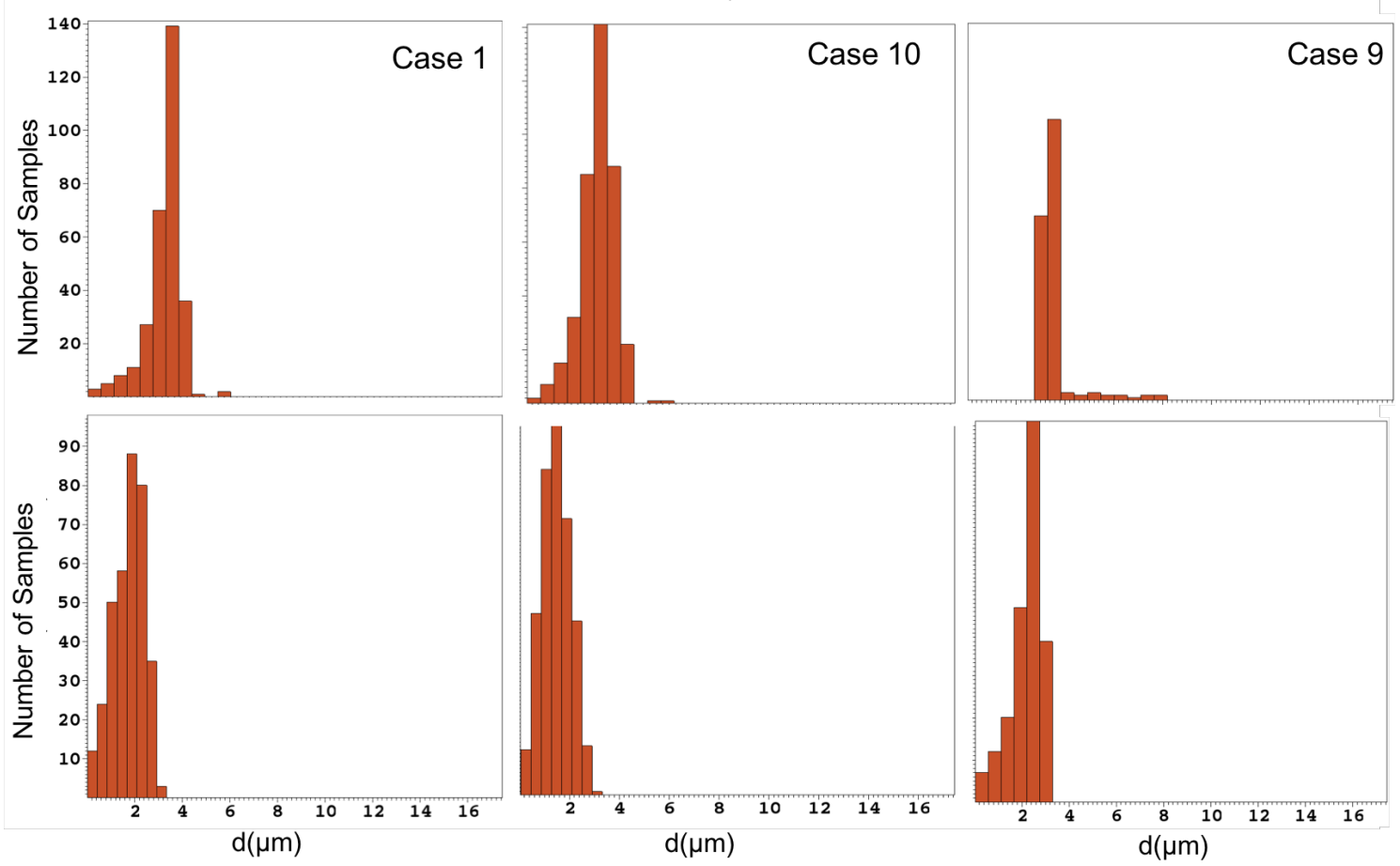

Figure 8. Number of droplets vs droplet sizes for two axial locations. Top raw is at $x=0.003 \mathrm{~m}$ (near-nozzle region) ant bottom row at $x=0.09 \mathrm{~m}$ (close to the tip of the spray). In all cases the PPS is 20 million and the samples were taken for $t=0.6 \mathrm{~ms}$.

\section{References}

[1] U. Azimov, N. Kawahara, and K. Tsuboi E. Tomita. Journal of Thermal Science and Technology, 5:238-251, 2010.

[2] A.G. Bailey, W. Balachandran, and T.J. Williams. Journal of Aerosol Science, 14(1):39 - 46, 1983.

[3] A. Banaeizadeh, A. Afshari A., H. Schock H., and F. Jaberi. International Journal of Heat and Mass Transfer, 60:781 - 796, 2013.

[4] G.M. Bianchi and P. Pelloni. SAE Technical Paper, 031999.

[5] C. Crua, S. Shoba, M. Heikal, M. Gold, and C. Higham. SAE paper 2010-01-2247, 2010.

[6] C. Cyril, R. Morgan, M. Heikal, and M.R. Gold. Fuel, 157:140 - 150, 2015.

[7] V. Granet, O. Vermorel, C. Lacour, B. Enaux, V. Dugue, and T. Poinsot. Combustion and Flame, 159(4):1562 1575, 2012.

[8] C.W Hirt and B.D Nichols. Journal of Computational Physics, 39(1):201 - 225, 1981.

[9] C.A. Idicheria and L. M. Pickett. SAE Technical Paper, 042007.

[10] F.P. Kärrholm. Numerical modelling of diesel spray injection, turbulence interaction and combustion. PhD thesis, Mech Eng, Goteborg, 2008.

[11] Sandia Laboratories. Engine Combustion Network, 2017 (accessed April 2017).

[12] T. M. Nguyen, F. Proch, I. Wlokas, and A. M. Kempf. Flow, Turbulence and Combustion, 97(1):191-230, 2016.

[13] F. Nicoud and F. Ducros. Flow, Turbulence and Combustion, 62(3):183-200, 1999.

[14] L.M. Pickett, J. Manin, C. L. Genzale, D.L. Siebers, M.P.B. Musculus, and C.A. Idicheria. SAE Int. J. Engines, 4:764-799, 042011.

[15] S. Quan, M. Dai, E. Pomraning, P.K. Senecal, K. Richards, S. Som, S. Skeen, J. Manin, and L. M. Pickett. SAE Int. J. Engines, 7:1054-1060, 042014.

[16] R.D. Reitz. Atomisation Spray Technology, 3:309-337, 1987.

[17] R. Scarcelli, K. Richards, E. Pomraning, P. K. Senecal, T. Wallner, and J. Sevik. SAE Technical Paper, 04 2016.

[18] M. Schmitt, C. E. Frouzakis, A. G. Tomboulides, Y. M. Wright, and K. Boulouchos. Proceedings of the Combustion Institute, 35(3):3069 - 3077, 2015.

[19] S.N. Soid and Z. Zainal. Energy, 36(2):724-741, 2011.

[20] S. Som, G. D'Errico, D. Longman, and T. Lucchini. SAE paper 2012-01-1263, apr 2012.

[21] S. Som, P.K. Senecal, and E. Pomraning. Comparison of rans and les turbulence models against constant volume diesel experiments. In ILASS, pages 1-10, 2012

[22] Q. Xue, S. Som, P. K. Senecal, and E. Pomraning. Atomization and Sprays, 23(10):925-955, 2013.

[23] S. Som Y. Pei, B. Hu. J. Energy Resour. Technol., 138(4):032205-032205-10, 2016.

[24] A. Yoshizawa and K. Horiuti. Journal of the Physics Society Japan, 54(8):2834-2839, 1985. 